PROCEEDINGS OF THE

AMERICAN MATHEMATICAL SOCIETY

Volume 140, Number 3, March 2012, Pages 1085-1096

S 0002-9939(2011)11214-0

Article electronically published on July 1, 2011

\title{
SECONDARY CHERN-EULER FORMS AND THE LAW OF VECTOR FIELDS
}

\author{
ZHAOHU NIE
}

(Communicated by Jianguo Cao)

\begin{abstract}
The Law of Vector Fields is a term coined by Gottlieb for a relative Poincaré-Hopf theorem. It was first proved by Morse and expresses the Euler characteristic of a manifold with boundary in terms of the indices of a generic vector field and the inner part of its tangential projection on the boundary. We give two elementary differential-geometric proofs of this topological theorem in which secondary Chern-Euler forms naturally play an essential role. In the first proof, the main point is to construct a chain away from some singularities. The second proof employs a study of the secondary Chern-Euler form on the boundary, which may be of independent interest. More precisely, we show by explicitly constructing a primitive that away from the outward and inward unit normal vectors, the secondary Chern-Euler form is exact up to a pullback form. In either case, Stokes' theorem is used to complete the proof.
\end{abstract}

\section{INTRODUCTION}

Let $X$ be a smooth oriented compact Riemannian manifold with boundary $M$. Throughout the paper we fix $\operatorname{dim} X=n \geq 2$ and hence $\operatorname{dim} M=n-1$. On $M$, we have a canonical decomposition

$$
\left.T X\right|_{M} \cong \nu \oplus T M,
$$

where $\nu$ is the rank 1 trivial normal bundle of $M$.

Let $V$ be a smooth vector field on $X$. We assume that $V$ has only isolated singularities, i.e., the set $\operatorname{Sing} V:=\{x \in X \mid V(x)=0\}$ is finite, and that the restriction $\left.V\right|_{M}$ is nowhere zero. Define the index $\operatorname{Ind}_{x} V$ of $V$ at an isolated singularity $x$ as usual (see, e.g., Hir76, p. 136]), and let Ind $V=\sum_{x \in \operatorname{Sing} V} \operatorname{Ind}_{x} V$ denote the sum of the local indices.

1.2. As an important special case, let $\vec{n}$ be the outward unit normal vector field of $M$, and $\vec{N}$ a generic extension of $\vec{n}$ to $X$. Then by definition,

$$
\text { Ind } \vec{N}=\chi(X) \text {, }
$$

where $\chi(X)$ is the Euler characteristic of $X$ (see, e.g., Hir76, p. 135]).

For a general $V$, let $\partial V$ be the projection of $\left.V\right|_{M}$ to $T M$ according to (1.1), and let $\partial_{-} V$ (resp. $\left.\partial_{+} V\right)$ be the restriction of $\partial V$ to the subspace of $M$, where $V$ points inward (resp. outward) to $X$. Generically $\partial_{ \pm} V$ have isolated singularities.

Received by the editors December 15, 2010.

2000 Mathematics Subject Classification. Primary 57R20, 57R25.

(C)2011 American Mathematical Society 
(A non-generic $V$ can always be modified by adding an extension to $X$ of a normal vector field or a tangent vector field to $M$.)

Using the flow along $-V$ and counting fixed points with multiplicities, we have the following Law of Vector Fields:

$$
\text { Ind } V+\operatorname{Ind} \partial_{-} V=\chi(X) \text {. }
$$

Naturally this is a relative Poincaré-Hopf theorem. It was first proved by Morse Mor29 and later on publicized by Gottlieb, who also coined the name "Law of Vector Fields".

One main purpose of this paper is to give two elementary differential-geometric proofs of this theorem (1.4).

In his famous proof Che44] of the Gauss-Bonnet theorem, Chern constructed a differential form $\Phi$ (see (2.7)) of degree $n-1$ on the tangent sphere bundle $S T X$, consisting of unit vectors in $T X$, satisfying the following two conditions:

$$
d \Phi=-\Omega,
$$

where $\Omega$ is the Euler curvature form of $X$ (pulled back to $S T X$ ), which is defined to be 0 when $\operatorname{dim} X$ is odd (see (2.11)), and

$$
\widetilde{\Phi_{0}}=\widetilde{d \sigma_{n-1}},
$$

i.e., the 0 th term $\widetilde{\Phi_{0}}$ of $\Phi$ is the relative unit volume form for the fibration $S^{n-1} \rightarrow$ $S T X \rightarrow X$ (see (2.8)). We call $\Phi$ the secondary Chern-Euler form.

Define $\alpha_{V}:\left.M \rightarrow S T X\right|_{M}$ by rescaling $V$, i.e., $\alpha_{V}(x)=\frac{V(x)}{|V(x)|}$ for $x \in M$. Then Chern's basic method Che45, §2], [BC65, §6] using the above two conditions and Stokes' theorem gives

$$
\int_{X} \Omega=-\int_{\alpha_{V}(M)} \Phi+\operatorname{Ind} V
$$

(see (2.12)). Applying (1.5) to the $\vec{n}$ and $\vec{N}$ in subsection 1.2 and using (1.3), one gets the following relative Gauss-Bonnet theorem in Che45]:

$$
\int_{X} \Omega=-\int_{\vec{n}(M)} \Phi+\operatorname{Ind}(\vec{N})=-\int_{\vec{n}(M)} \Phi+\chi(X) .
$$

Comparison of (1.5) and (1.6) gives

$$
\chi(X)=\operatorname{Ind} V+\int_{\vec{n}(M)} \Phi-\int_{\alpha_{V}(M)} \Phi .
$$

The following is our main result that identifies (1.7) with the Law of Vector Fields (1.4).

Theorem 1.8. The following formula holds:

$$
\int_{\vec{n}(M)} \Phi-\int_{\alpha_{V}(M)} \Phi=\operatorname{Ind} \partial_{-} V .
$$

A first proof of the above theorem is given in Section 3 The main point of this first proof is to construct, away from some singularities, a chain connecting $\alpha_{V}(M)$ to $\vec{n}(M)$ and then to apply Stokes' theorem.

A second proof of Theorem 1.8 to be given in Section 4 employs a study of the secondary Chern-Euler form on the boundary, i.e., when the structure group is 
reduced from $\mathrm{SO}(n)$ to $1 \times \mathrm{SO}(n-1)$. This study may be of some independent interest.

In more detail, the images $\vec{n}(M)$ and $(-\vec{n})(M)$ in $\left.S T X\right|_{M}$ are the spaces of outward and inward unit normal vectors of $M$. Define

$$
C S T M:=\left.S T X\right|_{M} \backslash(\vec{n}(M) \cup(-\vec{n})(M))
$$

( $C$ for cylinder) to be the complement. Also let $\pi:\left.S T X\right|_{M} \rightarrow M$ be the natural projection.

Theorem 1.10. There exists a differential form $\Gamma$ of degree $n-2$ on CSTM, such that after restricting to CSTM,

$$
\Phi-\pi^{*} \vec{n}^{*} \Phi=d \Gamma .
$$

The form $\Gamma$ is defined in (4.21), and the above theorem is proved right after that by utilizing Propositions 4.1 and 4.12 .

At the end of Section 4 we employ Stokes' theorem to give a second proof of Theorem 1.8, and hence of the Law of Vector Fields (1.4), using Theorem 1.10.

Remark 1.12. Unlike in Sha99] or [Nie11b], we do not assume that the metric on $X$ is locally a product near its boundary $M$. Therefore our results in this paper deal with the general case and generalize those in Nie11b.

Remark 1.13. We would like to emphasize the elementary nature of our approaches, in the classical spirit of Chern in [Che44,Che45]. Transgression of Euler classes has gone through some modern development utilizing Berezin integrals. The Thom class in a vector bundle and its transgression are studied in MQ86. This Mathai-Quillen form is further studied in BZ92 and BM06. For the modern developments, we refer the reader to the above references and two books, BGV92] and [Zha01], on this subject.

\section{Secondary Chern-Euler forms}

In this section, we review the construction, properties and usage of the secondary Chern-Euler form $\Phi$ in [Che44, which plays an essential role in our approaches.

Throughout the paper, $c_{r-1}$ denotes the volume of the unit $(r-1)$-sphere $S^{r-1}$. We also agree on the following ranges of indices:

$$
1 \leq A, B \leq n, 2 \leq \alpha, \beta \leq n-1,2 \leq s, t \leq n .
$$

The secondary Chern-Euler form $\Phi$ is defined as follows. Choose oriented local orthonormal frames $\left\{e_{1}, e_{2}, \cdots, e_{n}\right\}$ for the tangent bundle $T X$. Let $\left(\omega_{A B}\right)$ and $\left(\Omega_{A B}\right)$ be the $\mathfrak{s o}(n)$-valued connection forms and curvature forms for the LeviCivita connection $\nabla$ of the Riemannian metric on $X$ defined by

$$
\begin{gathered}
\nabla e_{A}=\sum_{B=1}^{n} \omega_{A B} e_{B}, \\
\Omega_{A B}=d \omega_{A B}-\sum_{C=1}^{n} \omega_{A C} \omega_{C B} .
\end{gathered}
$$

(In this paper, products of differential forms always mean "exterior products", although we omit the notation $\wedge$ for simplicity. Also, we closely follow Chern's notation and convention in Che44] and Che45. In particular we follow his convention in choosing the row and column indices in (2.2), which may not be the most 
standard.) Let the $u_{A}$ be the coordinate functions on $S T X$ in terms of the frames defined by

$$
v=\sum_{A=1}^{n} u_{A}(v) e_{A}, \quad \forall v \in S T X .
$$

Let the $\theta_{A}$ be the 1 -forms on $S T X$ defined by

$$
\theta_{A}=d u_{A}+\sum_{B=1}^{n} u_{B} \omega_{B A}
$$

For $k=0,1, \cdots,\left[\frac{n-1}{2}\right]$ (with [-] standing for the integral part), define degree $n-1$ forms on $S T X$ by

$$
\Phi_{k}=\sum_{A} \epsilon(A) u_{A_{1}} \theta_{A_{2}} \cdots \theta_{A_{n-2 k}} \Omega_{A_{n-2 k+1} A_{n-2 k+2}} \cdots \Omega_{A_{n-1} A_{n}},
$$

where the summation runs over all permutations $A$ of $\{1,2, \cdots, n\}$, and $\epsilon(A)$ is the sign of $A$. (The index $k$ stands for the number of curvature forms involved. Hence we have the restriction $0 \leq k \leq\left[\frac{n-1}{2}\right]$. This convention applies throughout the paper.) Define the secondary Chern-Euler form as

$$
\Phi=\frac{1}{(n-2) ! ! c_{n-1}} \sum_{k=0}^{\left[\frac{n-1}{2}\right]}(-1)^{k} \frac{1}{2^{k} k !(n-2 k-1) ! !} \Phi_{k}=: \sum_{k=0}^{\left[\frac{n-1}{2}\right]} \widetilde{\Phi_{k}} .
$$

The $\Phi_{k}$ and hence $\Phi$ are invariant under $\mathrm{SO}(n)$-transformations of the local frames and hence are intrinsically defined. Note that the 0th term

$$
\widetilde{\Phi_{0}}=\frac{1}{(n-2) ! ! c_{n-1}} \frac{1}{(n-1) ! !} \Phi_{0}=\frac{1}{c_{n-1}} d \sigma_{n-1}=\widetilde{d \sigma}_{n-1}
$$

is the relative unit volume form of the fibration $S^{n-1} \rightarrow S T X \rightarrow X$, since by (2.6),

$$
\Phi_{0}=\sum_{A} \epsilon(A) u_{A_{1}} \theta_{A_{2}} \cdots \theta_{A_{n}}=(n-1) ! d \sigma_{n-1}
$$

(see [Che44, (26)]).

Then [Che44, (23)] and Che45, (11)] prove that

$$
d \Phi=-\Omega,
$$

where

$$
\Omega= \begin{cases}0, & \text { if } n \text { is odd } \\ (-1)^{m} \frac{1}{(2 \pi)^{m} 2^{m} m !} \sum_{A} \epsilon(A) \Omega_{A_{1} A_{2}} \cdots \Omega_{A_{n-1} A_{n}}, & \text { if } n=2 m \text { is even }\end{cases}
$$

is the Euler curvature form of $X$.

Now we review Chern's basic method [Che45, §2], [BC65, §6] of relating indices, $\Phi$ and $\Omega$, using Stokes' theorem. Similar procedures will be employed twice later. Let $V$ be a generic vector field on $X$ with isolated singularities $\operatorname{Sing} V$. Let $B_{r}^{X}(\operatorname{Sing} V)$ (resp. $\left.S_{r}^{X}(\operatorname{Sing} V)\right)$ denote the union of small open balls (resp. spheres) of radii $r$ in $X$ around the finite set of points Sing $V$. Define $\alpha_{V}: X \backslash B_{r}^{X}(\operatorname{Sing} V) \rightarrow S T X$ 
by rescaling $V$. Then using (2.10) and Stokes' theorem, one proves (1.5) as

$$
\begin{aligned}
& \int_{X} \Omega=\lim _{r \rightarrow 0} \int_{\alpha_{V}\left(X-B_{r}^{X}(\operatorname{Sing} V)\right)} \Omega=\lim _{r \rightarrow 0} \int_{\alpha_{V}\left(X-B_{r}^{X}(\operatorname{Sing} V)\right)}-d \Phi \\
= & -\int_{\alpha_{V}(M)} \Phi+\lim _{r \rightarrow 0} \int_{\alpha_{V}\left(S_{r}^{X}(\operatorname{Sing} V)\right)} \Phi=-\int_{\alpha_{V}(M)} \Phi+\operatorname{Ind} V,
\end{aligned}
$$

where the last equality follows from the definition of the index and (2.8).

\section{First proof by CONSTRUCting a Chain}

In this section, we give a first proof of Theorem 1.8 by constructing a chain, away from Sing $\partial_{-} V$, connecting $\alpha_{V}(M)$ to $\vec{n}(M)$.

First proof of Theorem 1.8, By definition, Sing $\partial_{-} V$ consists of a finite number of points $x \in M$ such that $\alpha_{V}(x)=-\vec{n}(x)$. For $x \notin \operatorname{Sing} \partial_{-} V$, let $C_{x}$ be the unique directed shortest great circle segment pointing from $\alpha_{V}(x)$ to $\vec{n}(x)$ in $S T_{x} X$. With the obvious notation from before, let $U_{r}=M \backslash B_{r}^{M}\left(\operatorname{Sing} \partial_{-} V\right)$ denote the complement in $M$ of the union of open balls of radii $r$ in $M$ around Sing $\partial_{-} V$. Obviously its boundary $\partial U_{r}=-S_{r}^{M}\left(\operatorname{Sing} \partial_{-} V\right)$. Then

$$
\partial\left(\bigcup_{x \in U_{r}} C_{x}\right)=\bigcup_{x \in U_{r}} \partial C_{x}-\bigcup_{x \in \partial U_{r}} C_{x}=\vec{n}\left(U_{r}\right)-\alpha_{V}\left(U_{r}\right)+W_{r},
$$

with

$$
W_{r}:=\bigcup_{x \in S_{r}^{M}\left(\operatorname{Sing} \partial_{-} V\right)} C_{x} .
$$

Note the negative sign from the graded differentiation of chains in the second expression of (3.1). From (2.10) and (2.11), we have

$$
d \Phi=0 \text { on }\left.S T X\right|_{M},
$$

since even if $\operatorname{dim} X$ is even, $\left.\Omega\right|_{M}=0$ by a dimensional reason. (3.1), Stokes' theorem and (3.3) imply that

$$
\begin{aligned}
& \int_{\vec{n}(M)} \Phi-\int_{\alpha_{V}(M)} \Phi=\lim _{r \rightarrow 0}\left(\int_{\vec{n}\left(U_{r}\right)} \Phi-\int_{\alpha_{V}\left(U_{r}\right)} \Phi\right) \\
= & -\lim _{r \rightarrow 0} \int_{W_{r}} \Phi=-\lim _{r \rightarrow 0} \int_{W_{r}} \widetilde{\Phi_{0}},
\end{aligned}
$$

where the last equality follows from (2.7) and $\lim _{r \rightarrow 0} \int_{W_{r}} \widetilde{\Phi_{k}}=0$ for $k \geq 1$, since such $\widetilde{\Phi_{k}}$ 's in (2.6) involve curvature forms and do not contribute in the limit (see Che45, §2]). By (2.8), $\widetilde{\Phi_{0}}=\frac{1}{c_{n-1}} d \sigma_{n-1}$ is the relative unit volume form. We then compute the RHS of (3.4) using spherical coordinates.

3.5. At $\left.T X\right|_{M}$, we choose oriented local orthonormal frames $\left\{e_{1}, e_{2}, \cdots, e_{n}\right\}$ such that $e_{1}=\vec{n}$ is the outward unit normal vector of $M$. Therefore $\left(e_{2}, \cdots, e_{n}\right)$ are oriented local orthonormal frames for $T M$. Let $\phi$ be the angle coordinate on $\left.S T X\right|_{M}$ defined by

$$
\phi(v)=\angle\left(v, e_{1}\right)=\angle(v, \vec{n}),\left.\forall v \in S T X\right|_{M} .
$$


We have from (2.4)

$$
u_{1}=\cos \phi
$$

Let

$$
\begin{aligned}
p: C S T M=\left.S T X\right|_{M} \backslash(\vec{n}(M) \cup(-\vec{n})(M)) \rightarrow S T M ; v & \mapsto \frac{\partial v}{|\partial v|} \\
(\text { in coordinates })\left(\cos \phi, u_{2}, \cdots, u_{n}\right) & \mapsto \frac{1}{\sin \phi}\left(u_{2}, \cdots, u_{n}\right)
\end{aligned}
$$

be the projection to the equator $S T M$. By definition,

$$
p \circ \alpha_{V}=\alpha_{\partial V} \text { when } \partial V \neq 0 .
$$

Therefore the image of $W_{r}$ in (3.2) under the above projection is

$$
p\left(W_{r}\right)=\bigcup_{x \in S_{r}^{M}\left(\operatorname{Sing} \partial_{-} V\right)} p\left(C_{x}\right)=\bigcup_{x \in S_{r}^{M}\left(\operatorname{Sing} \partial_{-} V\right)} \alpha_{\partial V}(x)=\alpha_{\partial V}\left(S_{r}^{M}\left(\operatorname{Sing} \partial_{-} V\right)\right) .
$$

On $C_{x}$ for $x \in M$, the $\phi(3.6)$ ranges from $\phi\left(\alpha_{V}(x)\right)$ to 0 .

The relative volume forms $d \sigma_{n-1}$ of $\left.S^{n-1} \rightarrow S T X\right|_{M} \rightarrow M$ and $d \sigma_{n-2}$ of $S^{n-2} \rightarrow$ $S T M \rightarrow M$ are related by

$$
d \sigma_{n-1}=\sin ^{n-2} \phi d \phi p^{*} d \sigma_{n-2}+\text { terms involving } \omega_{1 s} \text { or } \Omega_{\alpha \beta}^{M} .
$$

(See (4.7) for the definition of the curvature forms $\Omega_{\alpha \beta}^{M}$. Also compare (4.18) when $k=0$ in view of (2.9). In the case of one fixed sphere and its equator, (3.10) without the extra terms is easy and follows from using spherical coordinates.) In the limit when $r \rightarrow 0$, the integrals of the terms involving $\omega_{1 s}$ or $\Omega_{\alpha \beta}^{M}$ are zero by the same reason as in the last step of (3.4).

Therefore, continuing (3.4) and using iterated integrals, we have

$$
\begin{aligned}
& \int_{\vec{n}(M)} \Phi-\int_{\alpha_{V}(M)} \Phi=-\lim _{r \rightarrow 0} \int_{W_{r}} \widetilde{\Phi_{0}}=-\frac{1}{c_{n-1}} \lim _{r \rightarrow 0} \int_{W_{r}} d \sigma_{n-1} \\
= & -\frac{1}{c_{n-1}} \lim _{r \rightarrow 0} \int_{\alpha_{\partial V}\left(S_{r}^{M}\left(\operatorname{Sing} \partial_{-} V\right)\right)}\left(\int_{\phi\left(\alpha_{V}(x)\right)}^{0} \sin ^{n-2} \phi d \phi\right) d \sigma_{n-2} \\
\stackrel{(1)}{=} & \frac{1}{c_{n-1}}\left(\int_{0}^{\pi} \sin ^{n-2} \phi d \phi\right) \lim _{r \rightarrow 0} \int_{\alpha_{\partial V}\left(S_{r}^{M}\left(\operatorname{Sing} \partial_{-} V\right)\right)} d \sigma_{n-2} \\
\stackrel{(2)}{=} & \frac{1}{c_{n-2}} \lim _{r \rightarrow 0} \int_{\alpha_{\partial V}\left(S_{r}^{M}\left(\operatorname{Sing} \partial_{-} V\right)\right)} d \sigma_{n-2} \stackrel{(3)}{=} \operatorname{Ind} \partial_{-} V .
\end{aligned}
$$

Here equality (1) uses

$$
\phi\left(\alpha_{V}(x)\right) \rightarrow \pi \text { for } x \in S_{r}^{M}\left(\operatorname{Sing} \partial_{-} V\right), \text { as } r \rightarrow 0,
$$

equality (2) uses the basic knowledge

$$
c_{n-1}=c_{n-2} \int_{0}^{\pi} \sin ^{n-2} \phi d \phi
$$

and equality (3) is by the definition of index.

Remark 3.13. The construction of the chain $\bigcup_{x \in U_{r}} C_{x}$ is reminiscent of the topological method [Mor29] of attaching $M \times I$ to $X$ and extending $\left.V\right|_{M}$ to a vector field on $M \times I$ whose value at $(x, t) \in M \times I$ is $(1-t) V(x)+t \vec{n}(x)$. 
Remark 3.14. The homology group $H_{n-1}\left(\left.S T X\right|_{M}, \mathbb{Z}\right) \cong \mathbb{Z} \oplus \mathbb{Z}$ has two generators as the image $\vec{n}(M)$ and a fiber sphere $S T_{x} M$ for $x \in M$ (see [Nie11a]). Our proof shows that as a homology class,

$$
\alpha_{V}(M)=\vec{n}(M)+\left(\text { Ind } \partial_{-} V\right) S T_{x} M
$$

\section{SECOND PROOF BY TRANSGRESSING $\Phi$}

In this section, we present a second transgression of the secondary Chern-Euler form $\Phi$ on $\left.C S T M \subset S T X\right|_{M}$ (1.9), leading to a proof of Theorem 1.10 and a second proof of Theorem 1.8 using that.

Recall the definition of the angle coordinate $\phi(3.6)$. Then $d \phi$ and $\frac{\partial}{\partial \phi}$ are a welldefined 1-form and a vector field on CSTM. We write $d$ for exterior differentiation on $C S T M$, and $\iota_{\frac{\partial}{\partial \phi}}$ for interior product with $\frac{\partial}{\partial \phi}$.

Proposition 4.1. On CSTM, let

$$
\Upsilon=\iota_{\frac{\partial}{\partial \phi}} \Phi
$$

Then the Lie derivative

$$
\mathcal{L}_{\frac{\partial}{\partial \phi}} \Phi=d \Upsilon
$$

Therefore

$$
\Phi-\pi^{*} \vec{n}^{*} \Phi=d \int_{0}^{\phi} \Upsilon d t
$$

Proof. (4.3) follows from the Cartan homotopy formula (see, e.g., KN63, Proposition I.3.10])

$$
\mathcal{L}_{\frac{\partial}{\partial \phi}} \Phi=\left(d \iota_{\frac{\partial}{\partial \phi}}+\iota_{\frac{\partial}{\partial \phi}} d\right) \Phi=d \Upsilon
$$

by (4.2) and $d \Phi=0$ (3.3).

(4.4) then follows by integration since $\pi^{*} \vec{n}^{*} \Phi$ corresponds to the evaluation of $\Phi$ at $\phi=0$ by the definition of $\phi(3.6)$, and we have for any fixed $\phi$ :

$$
\Phi-\pi^{*} \vec{n}^{*} \Phi=\int_{0}^{\phi} \mathcal{L}_{\frac{\partial}{\partial \phi}} \Phi d t=\int_{0}^{\phi} d \Upsilon d t=d \int_{0}^{\phi} \Upsilon d t .
$$

Now we calculate $\Upsilon$ explicitly. Since $\Phi$ (2.7) is invariant under $\mathrm{SO}(n)$-changes of local frames, we adapt an idea from [Che45] to use a nice frame for $\left.T X\right|_{M}$ to facilitate the calculations about $\Phi$ on $C S T M$. Choose $e_{1}$ as in subsection 3.5. For $v \in C S T M$, let

$$
e_{n}=p(v)
$$

as defined in (3.8). Choose $e_{2}, \cdots, e_{n-1}$ so that $\left\{e_{1}, e_{2}, \cdots, e_{n-1}, e_{n}\right\}$ is a positively oriented frame for $\left.T X\right|_{M}$. (Therefore we need $n \geq 3$ from now on, with the $n=2$ case being simple.) Then in view of (3.6),

$$
v=\cos \phi e_{1}+\sin \phi e_{n} .
$$

Let $\left(\Omega_{s t}^{M}\right)$ denote the curvature forms on $M$ of the induced metric from $X$. In view of (2.3),

$$
\begin{gathered}
\Omega_{s t}^{M}=d \omega_{s t}-\sum_{r=2}^{n} \omega_{s r} \omega_{r t} \\
\Omega_{s t}=\Omega_{s t}^{M}+\omega_{1 s} \omega_{1 t}
\end{gathered}
$$


Define the following differential forms on $S T M$, regarded to be pulled back to CSTM by $p$ (3.8), of degree $n-2$ :

$$
\begin{gathered}
\Phi^{M}(i, j)=\sum_{\alpha} \epsilon(\alpha) \omega_{1 \alpha_{2}} \cdots \omega_{1 \alpha_{n-2 i-j-1}} \Omega_{\alpha_{n-2 i-j} \alpha_{n-2 i-j+1}}^{M} \cdots \Omega_{\alpha_{n-j-2} \alpha_{n-j-1}}^{M} \\
\omega_{\alpha_{n-j} n} \cdots \omega_{\alpha_{n-1} n},
\end{gathered}
$$

where the summations run over all permutations $\alpha$ of $\{2, \cdots, n-1\}$. It is easy to check that these $\Phi^{M}(i, j)$ are invariant under $\mathrm{SO}(n-2)$-changes of the partial frames $\left\{e_{2}, \cdots, e_{n-1}\right\}$. Here the two parameters $i$ and $j$ stand for the numbers of curvature forms and $\omega_{\alpha n}$ 's involved. Define the following region of the indices $i, j$ :

$$
D_{1}=\{(i, j) \in \mathbb{Z} \times \mathbb{Z} \mid i \geq 0, j \geq 0,2 i+j \leq n-2\} .
$$

Then

$$
\Phi^{M}(i, j) \neq 0 \Rightarrow(i, j) \in D_{1} .
$$

Remark 4.10. Our choice of the letter $\Phi^{M}$ is due to the following special case when there are no $\omega_{1 \alpha}$ 's:

$$
\Phi^{M}(i, n-2 i-2)=\Phi_{i}^{M},
$$

where $\Phi_{i}^{M}$ are forms on $S T M$ defined by Chern [Che45]. Since we are considering the case of a boundary, we have the extra $\omega_{1 \alpha}$ 's in our more general forms.

Also note that the $\omega_{1 s}=0$ if the metric on $X$ is locally a product near the boundary $M$. Therefore a lot of our forms vanish in that simpler case as considered in [Nie11b.

We also introduce the following functions of $\phi$ (3.6), for non-negative integers $p$ and $q$ :

$$
\begin{gathered}
T(p, q)(\phi)=\cos ^{p} \phi \sin ^{q} \phi, \\
I(p, q)(\phi)=\int_{0}^{\phi} T(p, q)(t) d t .
\end{gathered}
$$

Proposition 4.12. We have the following concrete formulas:

$$
\Upsilon=\iota \frac{\partial}{\partial \phi} \Phi=\frac{1}{(n-2) ! ! c_{n-1}} \sum_{(i, j) \in D_{1}} a(i, j)(\phi) \Phi^{M}(i, j),
$$

$$
a(i, j)(\phi)=\sum_{k=i}^{\left[\frac{n-j}{2}\right]-1}(-1)^{n+j+k} \frac{(n-2 k-2) ! !}{2^{k} j !(n-2 k-j-2) ! i !(k-i) !} T(n-2 k-j-2, j)(\phi) .
$$

Proof. From (2.4), (2.5) and (4.6), we have

$$
\begin{gathered}
u_{1}=\cos \phi, u_{n}=\sin \phi, u_{\alpha}=0 ; \\
\theta_{1}=-\sin \phi\left(d \phi+\omega_{1 n}\right), \theta_{n}=\cos \phi\left(d \phi+\omega_{1 n}\right), \\
\theta_{\alpha}=\cos \phi \omega_{1 \alpha}-\sin \phi \omega_{\alpha n} .
\end{gathered}
$$

From (4.15), there are only two non-zero coordinates $u_{1}$ and $u_{n}$. Hence there are four cases for the positions of the indices 1 and $n$ in $\Phi_{k}$ (2.6):

(i) $n-2 k-1$ possibilities of $u_{1} \theta_{n}$; 
(ii) $2 k$ possibilities of $u_{1} \Omega_{\alpha n}$;

(iii) $n-2 k-1$ possibilities of $u_{n} \theta_{1}$;

(iv) $2 k$ possibilities of $u_{n} \Omega_{1 \alpha}$.

Only cases (i) and (iii) contribute $d \phi$ in view of (4.16), and hence we are only concerned with these two cases for the computation of $\Upsilon=\iota_{\frac{\partial}{\partial \phi}} \Phi$. Starting with (2.6), taking signs into consideration, by (4.15) and (4.16), by $\cos ^{2} \phi+\sin ^{2} \phi=1$, (4.17), (4.8) and the multinomial theorem, we have

$$
\begin{aligned}
\Phi_{k}= & (n-2 k-1)(-1)^{n} \cos ^{2} \phi\left(d \phi+\omega_{1 n}\right) \\
& \sum_{\alpha} \epsilon(\alpha) \theta_{\alpha_{2}} \cdots \theta_{\alpha_{n-2 k-1}} \Omega_{\alpha_{n-2 k} \alpha_{n-2 k+1}} \cdots \Omega_{\alpha_{n-2} \alpha_{n-1}} \\
& +(n-2 k-1)(-1)^{n} \sin ^{2} \phi\left(d \phi+\omega_{1 n}\right) \\
& \sum_{\alpha} \epsilon(\alpha) \theta_{\alpha_{2}} \cdots \theta_{\alpha_{n-2 k-1}} \Omega_{\alpha_{n-2 k} \alpha_{n-2 k+1}} \cdots \Omega_{\alpha_{n-2} \alpha_{n-1}} \\
& +\cdots \\
= & (-1)^{n}(n-2 k-1)\left(d \phi+\omega_{1 n}\right) \sum_{\alpha} \epsilon(\alpha)\left(\cos \phi \omega_{1 \alpha_{2}}-\sin \phi \omega_{a_{2} n}\right) \cdots \\
& \left(\cos \phi \omega_{1 \alpha_{n-2 k-1}}-\sin \phi \omega_{a_{n-2 k-1} n}\right)\left(\Omega_{\alpha_{n-2 k} \alpha_{n-2 k+1}}^{M}+\omega_{1 \alpha_{n-2 k}} \omega_{1 \alpha_{n-2 k+1}}\right) \cdots \\
& \left(\Omega_{\alpha_{n-2} \alpha_{n-1}}^{M}+\omega_{1 \alpha_{n-2}} \omega_{1 \alpha_{n-1}}\right) \\
& +\cdots \\
= & (-1)^{n}(n-2 k-1)\left(d \phi+\omega_{1 n}\right) \\
& \sum_{0 \leq i \leq k} \frac{(n-2 k-2) !}{j !(n-2 k-j-2) !} \cos ^{n-2 k-j-2} \phi(-\sin \phi)^{j} \frac{k !}{i !(k-i) !} \Phi^{M}(i, j) \\
& +\cdots \leq n \leq-2 k-2 \\
= & \sum_{\substack{0 \leq i \leq k \\
0 \leq}}(-1)^{n+2 k-2} \frac{(n-2 k-1) ! k !}{j !(n-2 k-j-2) ! i !(k-i) !} T(n-2 k-j-2, j)(\phi) \\
& \cdots\left(d \phi+\omega_{1 n}\right) \Phi^{M}(i, j)+\cdots
\end{aligned}
$$

From (2.7) and the above, we get (4.13) and the coefficients $a(i, j)(\phi)$ in (4.14), after some immediate cancellations.

Definition 4.19. For $(i, j) \in D_{1}$, define the following functions on $C S T M$ :

$$
\begin{aligned}
& A(i, j)(\phi)=\int_{0}^{\phi} a(i, j)(t) d t \\
(4.20)= & \sum_{k=i}^{\left[\frac{n-j}{2}\right]-1}(-1)^{n+j+k} \frac{(n-2 k-2) ! !}{2^{k} j !(n-2 k-j-2) ! i !(k-i) !} I(n-2 k-j-2, j)(\phi),
\end{aligned}
$$


in view of (4.14) and (4.11). Also define the differential form of degree $n-2$ on CSTM:

$$
\Gamma=\frac{1}{(n-2) ! ! c_{n-1}} \sum_{(i, j) \in D_{1}} A(i, j)(\phi) \Phi^{M}(i, j)
$$

Proof of Theorem 1.10. We just need to notice that $\Gamma=\int_{0}^{\phi} \Upsilon d t$ by Proposition 4.12 and use (4.4) in Proposition 4.1.

Remark 4.22. Our first proof of Theorem 1.10 was through very explicit differentiations. Write $\Phi=d \phi \Upsilon+\Xi$ in view of (4.2). We can compute $\Xi$ explicitly. After correctly guessing the $\Gamma$ in (4.21), we prove Theorem 1.10 by some differentiation formulas of differential forms in the spirit of Che45, and some induction formulas for the functions $I(p, q)(\phi)$ in (4.11) through integration by parts.

We finally arrive at

Second proof of Theorem 1.8. Let $B_{r}^{M}(\operatorname{Sing} \partial V)$ (resp. $\left.S_{r}^{M}(\operatorname{Sing} \partial V)\right)$ denote the union of small open balls (resp. spheres) of radii $r$ in $M$ around the finite set of points Sing $\partial V$. Then by $\partial V(x)=0 \Leftrightarrow \alpha_{V}(x)= \pm \vec{n}(x)$,

$$
\alpha_{V}\left(M \backslash B_{r}^{M}(\operatorname{Sing} \partial V)\right) \subset C S T M .
$$

By Theorem 1.10 and Stokes' theorem,

$$
\begin{aligned}
& \int_{\alpha_{V}(M)} \Phi-\int_{\vec{n}(M)} \Phi=\int_{\alpha_{V}(M)} \Phi-\pi^{*} \vec{n}^{*} \Phi=\lim _{r \rightarrow 0} \int_{\alpha_{V}\left(M \backslash B_{r}^{M}(\operatorname{Sing} \partial V)\right)} \Phi-\pi^{*} \vec{n}^{*} \Phi \\
= & \lim _{r \rightarrow 0} \int_{\alpha_{V}\left(M \backslash B_{r}^{M}(\operatorname{Sing} \partial V)\right)} d \Gamma=-\lim _{r \rightarrow 0} \int_{\alpha_{V}\left(S_{r}^{M}(\operatorname{Sing} \partial V)\right)} \Gamma \\
= & -\lim _{r \rightarrow 0} \int_{\alpha_{V}\left(S_{r}^{M}(\operatorname{Sing} \partial V)\right)} \frac{1}{(n-2) ! ! c_{n-1}} A(0, n-2)(\phi) \Phi^{M}(0, n-2),
\end{aligned}
$$

since all the other $A(i, j)(\phi) \Phi^{M}(i, j)$ in (4.21), for $(i, j) \in D_{1}$ and not equal to $(0, n-2)$, involve either curvature forms $\Omega_{\alpha \beta}^{M}$ or connection forms $\omega_{1 \alpha}$ and hence do not contribute in the limit when integrated over small spheres.

We have by (4.20) and (4.9),

$$
\begin{aligned}
& \frac{1}{(n-2) ! ! c_{n-1}} A(0, n-2)(\phi) \Phi^{M}(0, n-2) \\
& =\frac{1}{(n-2) ! ! c_{n-1}} \frac{(n-2) ! !}{(n-2) !} I(0, n-2)(\phi) \sum_{\alpha} \epsilon(\alpha) \omega_{\alpha_{2} n} \cdots \omega_{\alpha_{n-1} n} \\
& \quad=\frac{1}{c_{n-1}} I(0, n-2)(\phi) p^{*} d \sigma_{n-2}
\end{aligned}
$$

with $d \sigma_{n-2}$ being the relative volume form of $S^{n-2} \rightarrow S T M \rightarrow M$, since

$$
\sum_{\alpha} \epsilon(\alpha) \omega_{\alpha_{2} n} \cdots \omega_{\alpha_{n-1} n}=(n-2) ! p^{*} d \sigma_{n-2}
$$

in view of (4.5) and by comparison with (2.9). 
Continuing (4.23) and using (4.24), we have

$$
\begin{aligned}
& \int_{\alpha_{V}(M)} \Phi-\int_{\vec{n}(M)} \Phi \\
& =-\frac{1}{c_{n-1}} \lim _{r \rightarrow 0} \int_{\alpha_{V}\left(S_{r}^{M}\left(\operatorname{Sing} \partial_{+} V\right) \cup S_{r}^{M}\left(\operatorname{Sing} \partial_{-} V\right)\right)} I(0, n-2)(\phi) p^{*} d \sigma_{n-2} \\
& \stackrel{(1)}{=}-\frac{1}{c_{n-1}}\left[I(0, n-2)(0) \lim _{r \rightarrow 0} \int_{\alpha_{\partial V}\left(S_{r}^{M}\left(\operatorname{Sing} \partial_{+} V\right)\right)} d \sigma_{n-2} d \sigma_{n-2}\right] \\
& \quad+I(0, n-2)(\pi) \lim _{r \rightarrow 0} \int_{\alpha_{\partial V}\left(S_{r}^{M}\left(\operatorname{Sing} \partial_{-} V\right)\right)} d \sigma_{n-2} \\
& \stackrel{(2)}{=} \frac{1}{c_{n-2}} \lim _{r \rightarrow 0} \int_{\alpha_{\partial V}\left(S_{r}^{M}\left(\operatorname{Sing} \partial_{-} V\right)\right)} \stackrel{(3)}{=}-\operatorname{Ind} \partial_{-} V .
\end{aligned}
$$

Here equality (1) uses (3.9), (3.11) and the similar

$$
\phi\left(\alpha_{V}(x)\right) \rightarrow 0 \text { for } x \in S_{r}^{M}\left(\operatorname{Sing} \partial_{+} V\right) \text {, as } r \rightarrow 0 .
$$

In view of (4.11), we have

$$
I(0, n-2)(0)=0, I(0, n-2)(\pi)=\int_{0}^{\pi} \sin ^{n-2} \phi d \phi .
$$

Then equality (2) follows from (3.12). Equality (3) is by the definition of the index.

\section{REFERENCES}

[BGV92] Nicole Berline, Ezra Getzler, and Michèle Vergne, Heat kernels and Dirac operators, Grundlehren der Mathematischen Wissenschaften [Fundamental Principles of Mathematical Sciences], vol. 298, Springer-Verlag, Berlin, 1992. MR1215720 (94e:58130)

[BZ92] Jean-Michel Bismut and Weiping Zhang, An extension of a theorem by Cheeger and Müller, Astérisque 205 (1992), 235 pp. (English, with French summary). With an appendix by François Laudenbach. MR1185803 (93j:58138)

[BC65] Raoul Bott and S. S. Chern, Hermitian vector bundles and the equidistribution of the zeroes of their holomorphic sections, Acta Math. 114 (1965), 71-112. MR0185607 $(32: 3070)$

[BM06] J. Brüning and Xiaonan Ma, An anomaly formula for Ray-Singer metrics on manifolds with boundary, Geom. Funct. Anal. 16 (2006), no. 4, 767-837. MR2255381(2007i:58042)

[Che44] Shiing-shen Chern, A simple intrinsic proof of the Gauss-Bonnet formula for closed Riemannian manifolds, Ann. of Math. (2) 45 (1944), 747-752. MR0011027(6:106a)

[Che45] _ On the curvatura integra in a Riemannian manifold, Ann. of Math. (2) 46 (1945), 674-684. MR0014760 (7:328c)

[Hir76] Morris W. Hirsch, Differential topology, Graduate Texts in Mathematics, No. 33. Springer-Verlag, New York, 1976. MR 0448362 (56:6669)

[KN63] Shoshichi Kobayashi and Katsumi Nomizu, Foundations of differential geometry. Vol. I, Interscience Publishers, a division of John Wiley \& Sons, New York-London, 1963. MR 0152974 (27:2945)

[MQ86] Varghese Mathai and Daniel Quillen, Superconnections, Thom classes, and equivariant differential forms, Topology 25 (1986), no. 1, 85-110. MR836726 (87k:58006)

[Mor29] Marston Morse, Singular points of vector fields under general boundary conditions, Amer. J. Math. 51 (1929), no. 2, 165-178. MR1506710

[Nie11a] Zhaohu Nie, The secondary Chern-Euler class for a general submanifold, Canadian Mathematical Bulletin, published electronically on April 25, 2011. doi:10.4153/CMB2011-077-8 (2011). 
[Nie11b] _ On Sha's secondary Chern-Euler class, Canadian Mathematical Bulletin, published electronically on May 13, 2011. doi:10.4153/CMB-2011-089-1 (2011).

[Sha99] Ji-Ping Sha, A secondary Chern-Euler class, Ann. of Math. (2) 150 (1999), no. 3, 11511158. MR1740983 (2001g:57057)

[Zha01] Weiping Zhang, Lectures on Chern-Weil theory and Witten deformations, Nankai Tracts in Mathematics, vol. 4, World Scientific Publishing Co. Inc., River Edge, NJ, 2001. MR:1864735 (2002m:58032)

Department of Mathematics, Penn State Altoona, 3000 Ivyside Park, Altoona, PennSYLVANIA 16601

Current address: Department of Mathematics and Statistics, Utah State University, Logan, Utah 84322

E-mail address: znie@psu.edu 